\title{
PHASIM, an advanced phased array antenna software simulator
}

\author{
W.P.M.N. Keizer \\ TNO Physics and Electronics Laboratory \\ P.O. Box 96864, 2509 JG The Hague, Netherlands \\ keizer@fel.tno.nl
}

\begin{abstract}
A sophisticated phased array simulator software package for the design and analysis of planar phased array antennas is presented. This package can accurately simulate the far-field characteristics of a large variety of planar phased array configurations in both the frequency and time domain. The simulator is coded in MATLAB version 5.3. Using MATLAB, numerical engineering problems can be solved in a fraction of time of time required by programs coded in FORTRAN or C. This paper addresses an assessment of the program, discusses its use and advanced far-field analysis capabilities.
\end{abstract}

\section{INTRODUCTION}

Most present software packages for the calculation and analysis of the far-field behaviour of phased array antennas is written in traditional programming languages such as FORTRAN or C. However, the advent of commercially available computer algebra systems can greatly simplify the software coding of such programs due to the automatic manipulation of mathematical formulas provided by computer algebra.

In this paper the phased array software simulator PHASIM (PHased Array SIMulator) is described. It is a program for the analysis and design of planar phased array antennas of arbitrary geometry. PHASDM is coded in MATLAB Version 5.3. MATLAB (MATrix LABoratory) is a mathematical software tool that combines numeric and symbolic analysis with extended graphics and programming capabilities using a plain text proprietary language. Its matrix-oriented language is designed for efficient numerical, large-scale computation and data analysis and it has hundreds of built-in numerical functions.

PHASIM offers accurate, high speed computation of the far-field patterns of planar phased antennas, advanced far-field analysis capabilities and comprehensive 2D and 3D visualisation of the simulated results. Execution time is minimised by coding the program as matrix operations and using 2D FFT (Fast Fourier Transform) techniques for calculating the farfield patterns. The program operates with a state-of-theart GUI (Graphical User's Interface), provides extensive error checking of user data input, and is intuitive to use. This paper will address the main capabilities of the program, the computational approach and will present some typical results including their execution times. An earlier version of the program was described in [1]. Since then functionality of the program has been extended substantially. The latest version allows performing the far-field simulations in the frequency as well as in the time domain, and can deal with apertures split up in subarrays and with switcheable time delay units connected to a group of $T / R$ (Transmit/Receive) modules. The element lattice configurations, originally restricted to rectangular and triangular types, have been extended to skew ones, which may have random row or column displacements.

\section{MAIN CAPBILITIES}

The phased array simulator PHASIM can handle planar phased array antennas with the elements spaced in rectangular, triangular and skew lattice configurations. Skew lattices having random row or column displacements of the elements can be handled as well. The elements of the array are allowed to be grouped in subarrays. Scanning of the main beam can be modelled by either phase shifters or time delay circuits inside the $T / R$ modules including the option to use switcheable TDUs (Time Delay Units) connected to a group of T/R modules. The radiating elements may have isotropic, $\cos \left(\right.$ theta), $\cos (\text { theta })^{\wedge} \mathrm{N}$ or a dipole type radiating characteristics. For the array aperture the user can specify a circular, elliptical, rectangular or polygon shape.

In order to get low-sidelobe patterns, the program features 8 different amplitude tapers for sum patterns and 4 amplitude tapers for difference patterns. For the sum tapers the choice can be made between Uniform, Triangular, Cosine on a Pedestal, Gaussian, Blackman, Kaiser, Bessel and Taylor. For the difference patters, 
models are included for the tapers, odd Rectangular, odd Triangular, odd Taylor and Bayliss.

Apart from computing 2D far-field patterns, PHASIM can also generate high-resolution 1D far-field cuts. The program uses the results of the $2 \mathrm{D}$ far-field calculation to extract the directivity of the antenna, the main beam peak position, the 3-dB beamwidth in both the $u$ - and vplane and the rms sidelobe level. As an option PHASIM determines also the number of sidelobes and the distribution of their peak levels. For tracking antennas with monopulse capability the program has the ability to determine the tracking slope for both the azimuth and elevation plane.

Visualisation comprises also the element layout along the aperture including the grouping in sub-arrays, the location of defective elements and the connection of the switcheable TDUs to the elements. The element excitations, phase or time delay and amplitude can be displayed in 2D formats and $3 \mathrm{D}$ formats using pseudo contour or surface plots.

PHASIM can simulate various types of errors, such random phase and amplitude deviations in the aperture excitation, phase shifter or time delay quantisation, element failures randomly located or grouped in sectors, element location displacements and structural deformation of the aperture.

The program operates with an interactive Help Window, which gives access to an electronic on-line manual. This manual, coded as HTML (HyperText Markup Language) document, requires a Web browser to open it. PHASIM incorporates a state-of-the-art GUI and provides a high degree of user friendliness.

\section{COMPUTATIONAL APPROACH}

For arrays with periodic element lattice configurations, rectangular, triangular and skew (including the random row or column displacement) the computation of 2D far-field radiation pattern is always done by modified 2D FFT techniques performed on the element excitations. This approach reveals the $\mathrm{AF}$ (Array Factor), which multiplied with the element pattern gives the far-field pattern of the array under consideration. In case the elements are arbitrarily located, the $2 \mathrm{D}$ far-field patterns are computed by a fast brute force method based on direct summation of the radiating contributions of the array elements. All 2D farfield results are computed as a function of the direction cosine $u$ and $v$.

A 2D FFT is a very efficient and high-speed computational technique for numerical evaluation of the AF. Normally, a 2D FFT can only be applied when the array elements are arranged in a rectangular lattice.
However, in PHASIM this technique is extended to arrays using triangular or skew lattices. The earlier paper [1] provides information how FFT techniques can be applied to calculate the AF for triangular grids. For the present version of PHASIM a new FFT technique was developed which can also handle skew lattice configurations. This new FFT technique provides also more flexibility with respect to the spacing between the far-field directions. With conventional FFTs the spacing between the far-field directions is fully determined by the aperture element spacing, the frequency of operation and the size of the FFT. With the new FFT to calculate the $\mathrm{AF}$, the spacing between the far-field directions is no longer subjected to any restriction. Another advantage of this new technique is its zoom-in capability, which allows computing the 2D far-field with a high density of the far-field directions over a limited spatial region without using a large size FFT.

The program can compute high-resolution far-field pattern cuts in the u- or v-direction or in spherical coordinates Phi and Theta. Computation of the pattern cuts for a fixed u- or v-direction relies on a hybrid approach consisting of the brute force method in combination with a large size (8192 points) 1D FFT. These kind of farfield cuts can be computed for any fixed value of $u$ or $v$ between -1 and 1 . The pattern cuts for the spherical coordinates $\mathrm{Phi}$ and Theta are derived from the 2D farfield results using an interpolation scheme based on a built-in MATLAB 2D interpolation M-function.

In order to optimise execution speed, the program was mainly coded as matrix operations; in particular the far-field computations and the data visualisation parts were programmed in this way. For MATLAB this is the favourite approach since it is matrix oriented programming language. This results in a very compact code compared to the coding in FORTRAN or $\mathrm{C}$ which is therefore also much more easier to debug or to maintain.

\section{SIMULATION EXAMPLES}

Figs 1-2 show typical simulation results obtained with PHASIM. Both figures deal with the same phased array configuration, which operates with time-delay steering for scanning the beam. Two types of switcheable time delay devices are specified. At the element level a 200 ps timeshifter is applied with 6-bit resolution in combination with 103 switcheable TDUs located at the feed level. The TDUs have a 10 bit resolution and a maximum time delay range of $5000 \mathrm{ps.}$ The array operates with 3407 radiating elements. Each TDU feeds 15 rows and 9 columns of elements. The plot 
at the top of Fig. 1 represents the far-field sum pattern on transmit of a circular phased array antenna with a triangular lattice arrangement of the elements. In this example the beam is scanned to Theta $=30 \mathrm{deg}$ and Phi $=40$ deg. A Blackman taper was selected for lowsidelobe performance. However, since the beam steering is done with 6-bit timeshifters, quantization lobes due to the finite time delay resolution of the timeshifters and TDUs, corrupt the low sidelobe performance. The bottom plot of Fig. 1 reveals the peak sidelobe performance of the array antenna. Data on the beam position, antenna directivity and gain, 3-dB beamwidth and rms sidelobe level is depicted in the right part of Fig. 1.

Fig. 2 demonstrates the advantage of using time delay steering for scanning the main beam. As can be seen from the top plot, the angular position of the main beam does not vary with signal frequency. The bottom plot shows for the fixed far-field direction Theta $=30$ $\mathrm{deg}$ and $\mathrm{Phi}=40 \mathrm{deg}$ the behaviour of the array gain and the array group delay over the frequency band 9-12 $\mathrm{GHz}$.

PHASIM, running on a $\mathrm{PC}$ with a $500 \mathrm{MHz}$ Pentium III processor, computes a $2 \mathrm{D}$ far-field pattern for $256 * 256$ cosine directions in less than $0.3 \mathrm{sec}$. The far- field analysis, revealing directivity, peak direction, 3-dB beamwidth for both the $u$ - and $v$ plane including the rms sidelobe level takes a about the same time. The duration of the peak sidelobe analysis depends on the number of sidelobes and is usually done within 1 second including the plotting of the peak sidelobe histogram.

\section{CONCLUSIONS}

A sophisticated phased array simulator written in MATLAB 5.3 has been developed at TNO-FEL. The simulator can deal with various aperture shapes, a number of periodic element lattice configurations and various aperture tapers both for sum and difference patterns. High speed computation is obtained by coding the program as matrix or vector operations.

\section{REFERENCES}

[1] Keizer, W.P.M.N., "PHASIM, a sophisticated phased array antenna software simulator implemented in MATLAB 5.2", IEEE AP-S International Symposium 1999, Orlando, pp. 25082511. 


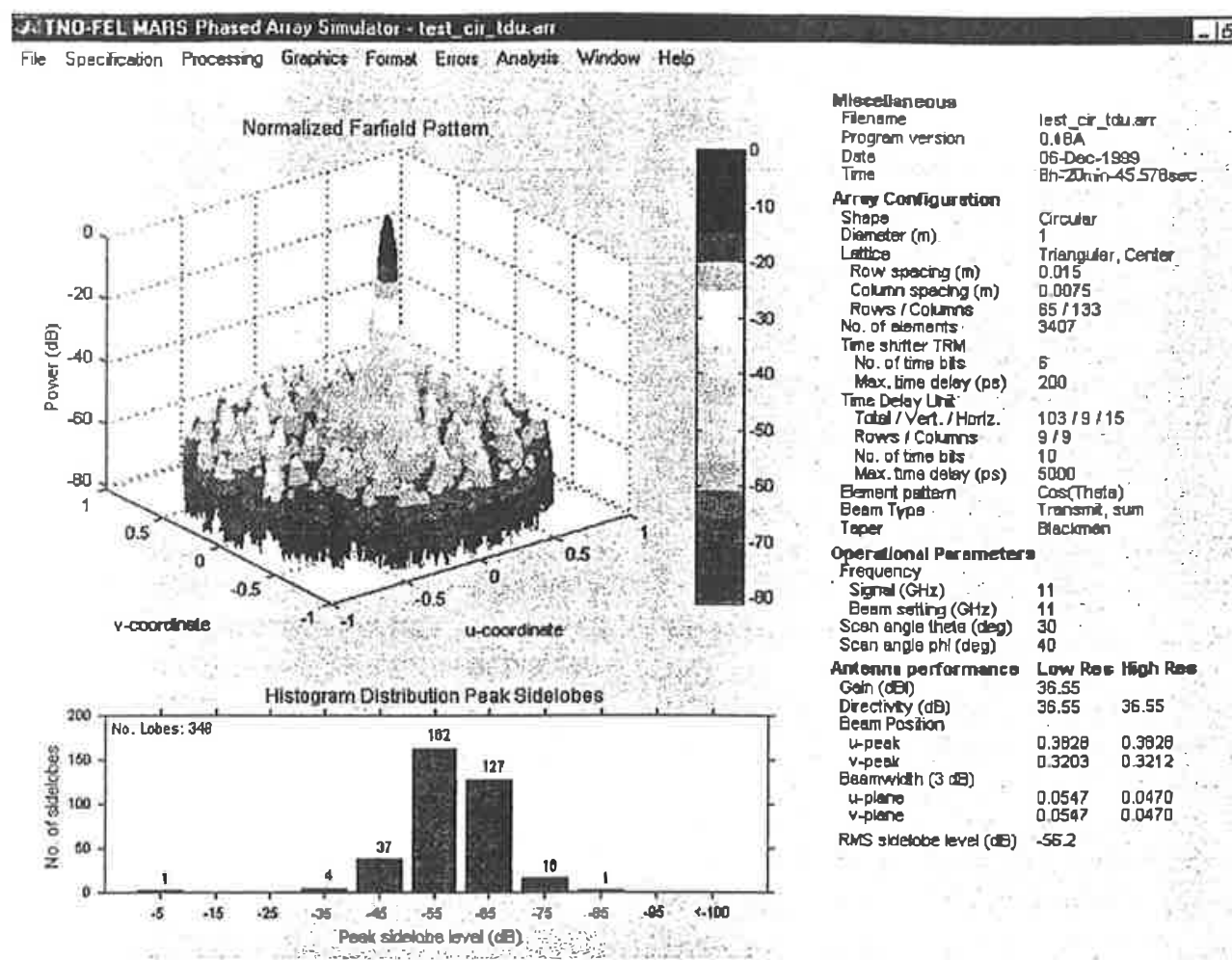

Fig. 1. Far-field results of a circular phased array at $11 \mathrm{GHz}$ with the main beam scanned at Theta $=30$ deg and $\mathrm{Phi}=40 \mathrm{deg}$. Beam scanning is realised by time delay steering.

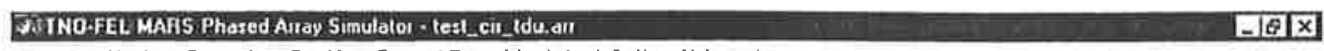

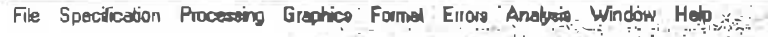
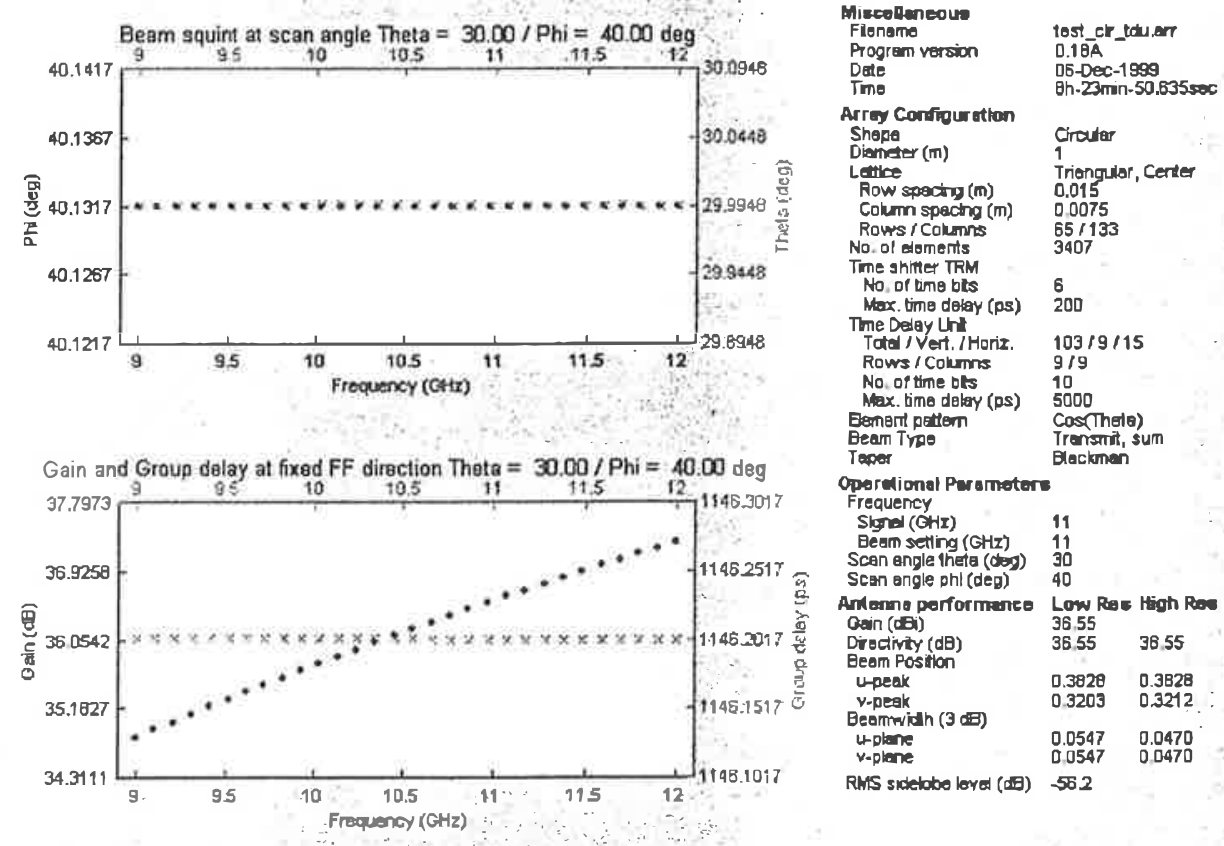

Fig.2 Beam squint behaviour and transfer characteristic (gain and group delay) of the same phased array over the frequency band $9-12 \mathrm{GHz}$ at the scan direction Theta $=30 \mathrm{deg}$ and Phi $=40 \mathrm{deg}$. 
FLIT od Bogant

\section{International Symposium on ANTENNAS FOR RADAR EARTH OBSERVATION}

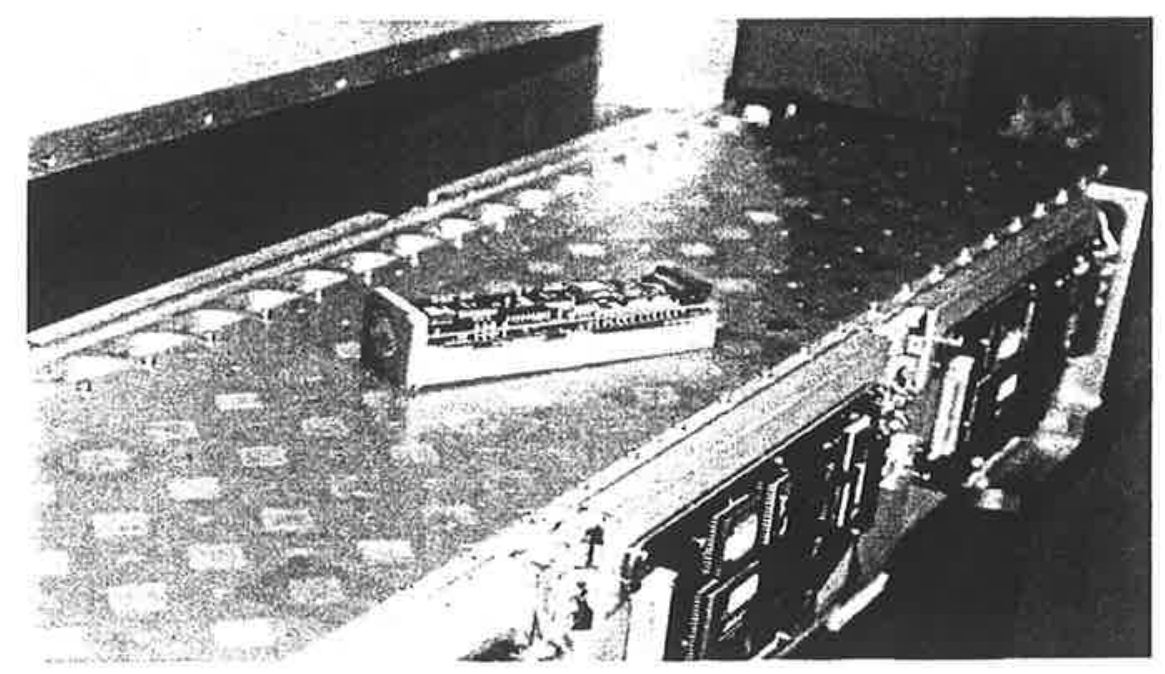

\section{Symposium Proceedings}

Held on the occasion of the inauguration of Prof. P. Hoogeboom, at Delft University of Technology, the Netherlands, 8-9th June 2000.

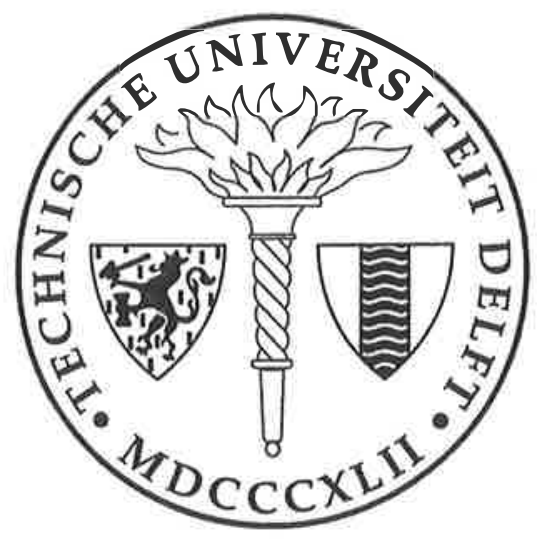

$8-9^{\text {th }}$ June 2000

Delft University of Technology

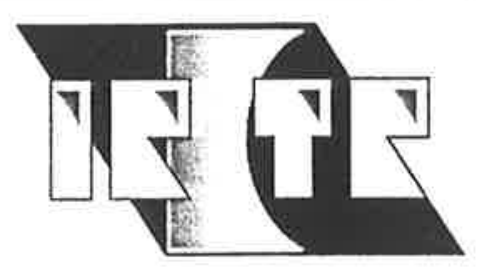

International Research Centre for Telecommunications-Transmission and Radar 